\title{
Anxiety and depression in rheumatologic diseases: the relevance of diagnosis and management
}

\author{
R. Torta, F. Pennazio, V. leraci \\ Clinical and Oncological Psychology Unit, University of Turin, Italy
}

\section{SUMMARY}

The high prevalence of emotional disorders (anxiety, chronic stress, mood depression) in patients with pain during rheumatologic diseases (particularly fibromyalgia) is closely related to the common pathogenic mechanisms concerning emotions and pain. Therefore a prompt identification of any psychic component of pain, also by means of specific tools, is a must, because it can require an adjustment of the therapeutic approach by combining both an analgesic treatment and antidepressants and/or psychotherapeutic strategies.

Key words: Emotional disorders, Antidepressants, Psychotherapeutic strategies.

Reumatismo, 2014; 66 (1): 92-97

\section{INTRODUCTION}

T. he prevalence of anxiety and depressive symptoms in rheumatologic diseases is well known and accounts for $93 \%$ and $94 \%$ of patients affected by lupus erythematous and rheumatoid arthritis respectively. Patients report symptoms of cognitive impairment in $66 \%$ of cases, fatigue in $40 \%$ and sleep disorders in $72 \%$ (1-3). In our pool of patients with fibromyalgia (FM), mood depression is present in $60 \%$ of them, anxiety in $52 \%$ and chronic stress in $72 \%$ (4).

The pathogenesis of mood, anxiety, sleep disorders and pain in rheumatic diseases, according to the bio-psycho-social model, is multifactorial and shows several overlapping factors, such as genetic factors, changes in the central nervous system (CNS) and autonomic nervous system, inflammatory alterations and environmental factors (5). Pain in itself is correlated with anxiety, mood depression and chronic stress (5).

There is a significant interaction between the neurobiological factors underlying emotional components, rheumatologic diseases and their relationship. A genetic vulnerability can modulate the response to stress and somatic injuries, acting at a cel- lular/subcellular level (for example modifying intracellular signaling, gene transcription, neurotrophic response) that, in turn, influences the networks (causing for example the shift from a functional dysregulation of neural circuits to structural changes) (6).

These alterations can lead to modifications of endocrine, autonomic, immune systems that induce both neuropsychiatric and somatic systemic symptoms. In this paper we will discuss the relationships among chronic stress, mood and pain, their clinical relevance and the need for a correct management of such aspects, in order to improve the quality of life of patients and the course of their disease.

\section{DISTRESS, MOOD AND PAIN}

In our experience, $72 \%$ of outpatients with FM show signs of distress (4). This is the result of the screening that we carry out with a fast and easy tool called distress thermometer (DT). The DT is a visual analog instrument that asks the respondent to rate his/her level of distress in the past week, on a scale from 0 (no distress) to 10 (extreme distress). The tool is complemented with a 
problem list (PL) of 34 problems, grouped into 5 categories (practical problems, family problems, emotional problems, spiritual/ religious concerns, and physical problems) that are rated in a yes/no format.

The validity of the DT has been confirmed in many countries, even in the oncologic populations, and recently also in Italy. Grassi et al. (7) have assessed the validity and acceptance of the DT by 1108 patients [329 men (30\%) and 779 (70\%) women; mean age, $53.4+9.3$ years] in a nationwide validation study, carried out in 38 Italian cancer centers, which are representative of the different regions of the country.

In this study a cut-off score of 4 has demonstrated that $47 \%$ of patients can be affected by distress, with a tendency to overestimate caseness as measured by the hospital anxiety and depression scale (HADS) and brief symptom inventory (BSI-18) (33\% and $38 \%$ respectively), whereas a more conservative cut-off score of 5 indicated a significant distress in $33 \%$ of patients with cancer.

The single item DT compares favorably with longer measures that are used to screen for distress and, when the DT is combined with the PL, it makes it easier to identify cancer-related problems. In a fluorodeoxyglucose-positron emission tomography study of the relationship between DT and brain metabolism in cancer patients, we have recently confirm that the DT correlates with brain areas that are typically involved in the stress response. In fact hypothalamic metabolism was found to be the best predictor of distressed patients according to DT scores (8).

In conclusion, a brief screening tool like the DT is a simple and effective screening instrument for detecting distress in Italian patients. Interestingly a genetic component, such as the presence of the short allele for the serotonin transporter (5HTTPR), can significantly contribute to poor resilience to stress (9).

Therefore this tool can help identify a subgroup of patients that can be at a higher risk of developing a pathological distress in the course of the disease. One of the major biological components in the connection between stress, mood and pain is the hyperactivity of the hypothalamus-pituitaryadrenal (HPA) axis, with ensuing hypercortisolism, glucocorticoid resistance, sympathetic over-activity and increased release of pro-inflammatory cytokines $[$ e.g. tumor necrosis factor (TNF)- $\alpha$, interleukin (IL)-1, IL-6] $(10,11)$.

The hormonal response of the HPA axis starts with an increase of the cortisol releasing hormone $(\mathrm{CRH})$ that activates a chain of other hormones, such as adrenocorticotropic hormone and cortisol. $\mathrm{CRH}$ in itself is able to induce bio-behavioral typical stress responses, such as an increase in heart rate and respiration rate, an increase in blood pressure and blood sugar level, and a decrease in sexual activity and food intake. Moreover CRH causes a sympathetic activation and a parasympathetic inhibition.

All these responses are related to the release of CRH1, or to the inhibition of CHR2, which are linked to the pro- and antinoceptive activity in the amygdala neurons (12). The increase of stress-related pro-inflammatory cytokines is associated with several clinical symptoms, such as pain, fatigue, cognitive impairment, mood depression, and anxiety (13). For these reasons, distress has to be considered as the sixth vital sign and must be monitored in order to identify and treat stressed patients (14).

The concept of neuroinflammation, which is associated with the CNS response to prolonged stress, is also supported by microglial activation that induces a glutamatergic increase, due to a reduced glutamatergic reuptake and an increased glutamatergic release from astrocytes, which in turn favor neuronal stress-induced excitotoxicity (15).

\section{MOOD DEPRESSION AND PAIN}

Depressive disorders are common and frequently associated with chronic pain in sort of vicious circle. The depressive mood reduces the pain threshold and increases pain perception both emotionally and cog- 
nitively, while chronic pain first induces strained relationships, reduces perceived self-efficacy, increases disability, causes first demoralization, then true depression $(5,16)$.

It is important to consider that at times in a depressed patient a cluster of unexplained physical symptoms may reflect symptoms of mood depression. In a national sample of Italian psychiatric outpatients a large number of unexplained somatic symptoms were associated with more severe depression and higher rates of misdiagnosis and inappropriate treatments.

In particular $41.3 \%$ of depressed patients had 1-5 unexplained symptoms and $42.7 \%$ had more than 5. Fatigue was the most commonly observed unexplained somatic symptom, being present in $53 \%$ of patients (17).

Hence, in this perspective, many symptoms (for example pain, fatigue, cognitive impairment) have to be seen from two pathogenic points of view. On one hand these symptoms are related to the somatic disease itself (for example fibromyalgia), and, on the other, they have a component which originates directly from mood depression. Consequently the therapeutic approach must face both pathogeneses: for example treating pain, when necessary, with both analgesic and psychopharmacologic/psychological strategies.

As to distress, also in diagnosing depression several fast and easy tools can be used in a first step screening. To this aim our group recently has validated the HADS, a self-reported simple screening scale, which requires a few minutes for compilation and scoring as against the Montgomery-Asberg depression rating scale, which is a widespread and more complex instrument based on a semi-structured clinician-rated scale, that requires about 15 minutes for administration and scoring by a trained clinician, depending on the severity of the symptoms and the difficulty of the interview (18).

The HADS is a 14-item (rated 0-3) self-report scale with two seven-item depression and anxiety subscales. The total HADS depression score ranges from 0 (absence of depression) to 21 (severe depression). For the purpose of screening, if a patient scores a cut-off $\geq 8$, he/she will attend a subsequent semi-structured clinical interview in order to confirm and improve the diagnosis.

Importantly the HADS depression subscale does not contain items related to the somatic components of mood depression, but only to the hedonic and volitional aspects of mood so as to avoid the bias caused by misunderstanding somatic components with emotional ones.

The frequent association between pain and depressed mood can be explained by a common biological background, which is, at the same time, involved in the modulation of both painful and emotional experience (16). In this context, several pathways are connected, such as those of neurotransmitters, hormones and cytokines.

A decrease in serotonin (5HT) and norepinephrine (NE), particularly in the limbic areas, is related to the monoaminergic hypothesis of depressive disorders. Furthermore a similar reduction occurs simultaneously in areas of the descendent inhibitory system, such as the periacqueductal griseum. As a result, the neurotransmitter deficit causes simultaneously both a bad mood and an increase in pain (5).

Also an alteration of the immune system, such as an increase of pro-inflammatory cytokines, can be related directly and indirectly to both depressive disorders and pain. The participation of cytokines in neurogenic inflammation and sickness behavior is largely documented: for example cytokines seem to play an important role in sensitizing deep tissue nociceptors of chronic widespread pain patients, and the combination of peripheral impulse input and increased central pain sensitivity may be responsible for widespread chronic pain disorders (19).

On the other hand, also neuroimaging studies emphasize the relationship between inflammatory states and depressive disorders. Using functional magnetic resonance imaging in rheumatoid arthritis (RA) patients, Schweinhardt et al. (20) have suggested that the medial prefrontal cortex plays an important role in mediating the relationship between depressive symptoms 
and clinical pain severity in RA, possibly by engaging brain areas that are important for the processing of affective components. Furthermore, there is prospective longitudinal evidence concerning the emergence of mood symptoms in response to chronic immune inflammatory activation (21). Elevated immune-inflammatory signaling is therefore a relevant mechanism in the pathogenesis of mood disorders (15), as also confirmed by the fact that an additional treatment with anti-inflammatory agents can sometimes increase the therapeutic efficacy of antidepressants (22). The proinflammatory cytokines (mainly IL2, IL6, $\mathrm{TNF} \alpha$ ) can interfere with mood in several ways: through the increase of $\mathrm{CRH}$ and the global activation of the HPA axis; by glucocorticoid resistance due to a decrease of GR expression; by a reduction of T3; by the excitoxicity due to the glutamate increase at microglial and astroglial level (15).

A very intriguing mechanism is the activity of the metabolic enzymeindoleamine 2,3-dioxygenase (IDO), which breaks down tryptophan into kynurenine. An increase in pro-inflammatory cytokines up-regulates IDO, thus moving the tryptophan from the serotonin pathway to the kynurenine pathway, and reducing 5HT production with consequent depressive disorders (23). On the other hand, cytokines, such as TNF $\alpha$ and IL1, play also an important role in sleep regulation. In normal humans and in multiple disease states, plasma levels of TNF $\alpha$ co-vary with the electroencephalogram slow wave activity and sleep propensity.

Many of the symptoms induced by sleep loss, such as sleepiness, fatigue, poor cognition, and enhanced sensitivity to pain are present in patients with $\mathrm{FM}$, and can be elicited by injecting exogenous $\mathrm{TNF} \alpha$ or IL (24).

Another important aspect is the interaction between pro-inflammatory cytokines and the neurotrophin brain-derived neurotropic factor (BDNF), in both depressive disorders and pain. From the clinical point of view, altered BDNF levels in the fibromyalgic syndrome suggest that BDNF is involved in the sensitization process, prob- ably through its effects on neuronal plasticity (25). Nevertheless, an involvement of neurotropic factors in their activity on pain is still debated (6).

Another noteworthy aspect concerning the relationship between mood and pain is the clinical observation that pain could be a symptom of depression (5).

Depressive disorders are actually related to a spectrum of symptoms, including affection (dysphoric mood, irritability, loss of interest and pleasure), cognition (impaired memory, concentration and decision-making, cognitive distortion), behavior (coping styles and psychomotor changes), but also somatic symptoms, such as alterations in appetite, weight, sleep patterns, sexual desire, increased fatigue, and chronic pain (headaches, back pain, visceral pain, etc.). In several patients an increasing number of unexplained physical symptoms are directly related to the depression severity (17).

\section{PAIN AND MOOD TREATMENT IN RHEUMATOLOGIC DISEASES}

Several classes of drugs are used in pain treatment, according to pain severity and World Health Organization guidelines (26): non-steroidal anti-inflammatory drugs, weak and strong opioids. If necessary, these classes can be associated with the so-called adjuvants, a wide class of pharmacological compounds, mainly antidepressants, anticonvulsants, and steroids. Moreover, the class of choice in anxiety, mood and chronic stress disorders is undoubtedly represented by antidepressants (ADs), which are drugs with a broad spectrum of clinical activities (27). ADs relieve pain through various mechanisms, which also have effects on depressive disorders (16), namely they increase 5HT and NE, neutralize the $\mathrm{CRH}$ activation and consequently reduce cytokine hyper-production, and increase neurotropic factors $(27,28)$.

On the other hand, cognitive and emotional components of pain widely justify the psychotherapeutic approach to pain perception. 
In this respect, several psychotherapeutic and support techniques, such as cognitive behavioral therapy, dynamically-oriented therapy, hypnosis, relaxation training, supportive psychotherapy and behavioral therapy, can be employed to address the psychological and social features associated with and contributing to pain. It is very important is to consider that these treatments do not exclude each other, but they can used in combination to effectively address different patients' needs and offer benefits in terms of both mood improvement and pain management (29).

\section{REFERENCES}

1. Abad VC, Sarinas PS, Guilleminault C. Sleep and rheumatologic disorders. Sleep Med Rev. 2008; 12: 211-28.

2. Omachi TA. Measures of sleep in rheumatologic diseases: Epworth Sleepiness Scale (ESS), Functional Outcome of Sleep Questionnaire (FOSQ), Insomnia Severity Index (ISI), and Pittsburgh Sleep Quality Index (PSQI). Arthritis Care Res (Hoboken). 2011; 63: S287-96.

3. Lisitsyna TA, Zeltyn' AE, Vel'tishchev DIu, Kovalevskaia OB, Seravina OF, Novikova DS, et al. [Cognitive impairment and anxiety-depressive disorders in patients with rheumatoid arthritis]. Zh Nevrol Psikhiatr Im S S Korsakova. 2012; 112: 96-103. [Article in Russian].

4. Castelli L, Tesio V, Colonna F, Molinaro S, Leombruni P, Torta R, et al. Alexithymia and psychological distress in fibromyalgia: prevalence and relation with quality of life. Clin Exp Rheumatol. 2012; 30: 70-7.

5. Torta RG, Ieraci V. Depressive disorders and pain: a joint model of diagnosis and treatment. J Pain Relief. 2013; S2: 1-14

6. Vanelderen P, Rouwette T, Kozicz T, Roubos E, Van Zundert J, Heylen R, et al. The role of brain-derived neurotrophic factor in different animal models ofneuropathic pain. Eur J Pain. 2010; 14: 473.

7. Grassi L, Johansen C, Annunziata MA, Capovilla E, Costantini A, Torta R, et al. Italian Society of Psycho-Oncology Distress Thermometer Study Group. Screening for distress in cancer patients: a multicenter, nationwide study in Italy. Cancer. 2013; 19: 1714-21.

8. Baudino B, D'Agata F, Caroppo P, Castellano G, Cauda S, Torta R, et al. The chemotherapy long-term effect on cognitive functions and brain metabolism in lymphoma patients. Q J Nucl Med Mol Imaging. 2012; 56: 559-68.

9. Hammen C, Brennan PA, Keenan-Miller D,
Hazel NA, Najman JM. Chronic and acute stress, gender, and serotonin transporter geneenvironment interactionspredicting depression symptoms in youth. J Child Psychol Psychiatry. 2010; 51: 180-7.

10. Thornton LM, Andersen BL, Blakely WP. The pain, depression, and fatigue symptom cluster in advanced breast cancer: covariation with the hypothalamic-pituitary-adrenal axis and the sympathetic nervous system. Health Psychol. 2010; 29: 333-7.

11. Zunszain PA, Anacker C, Cattaneo A, Carvalho LA, Pariante CM. Glucocorticoids, cytokines and brain abnormalities in depression. Prog Neuropsychopharmacol Biol Psychiatry. 2011; 35: 722-9.

12. Aguilera G, Nikodemova M, Wynn PC, Catt KJ. Corticotropin releasing hormone receptors: two decades later. Peptides. 2004; 25 : 319-29.

13. Felger JC, Lotrich FE. Inflammatory cytokines in depression: neurobiological mechanisms and therapeutic implications. Neuroscience. 2013; 246: 199-229.

14. Bultz BD, Johansen C. Screening for distress, the 6th vital sign: where are we, and where are we going? Psychooncology. 2011; 20: 569-71.

15. Krishnadas R, Cavanagh J. Depression: an inflammatory illness? J Neurol Neurosurg Psychiatry. 2012; 83: 495-502.

16. Torta RG, Munari J. Symptom cluster: depression and pain. Surg Oncol. 2010; 19: 155-9.

17. Perugi G, Canonico PL, Carbonato P, Mencacci C, Muscettola G, Torta R, et al. Come To Me Study Group. Unexplained somatic symptoms during majordepression: prevalence and clinical impact in a national sample of Italianpsychiatric outpatients. Psychopathology. 2011; 44: 116-24.

18. Castelli L, Binaschi L, Caldera P, Mussa A, Torta R. Fast screening of depression in cancer patients: the effectiveness of the HADS. Eur J Cancer Care (Engl). 2011; 20: 528-33.

19. Staud R. Peripheral pain mechanisms in chronic widespread pain. Best Pract Res Clin Rheumatol. 2011; 25: 155-64.

20. Schweinhardt P, Kalk N, Wartolowska K, Chessell I, Wordsworth P, Tracey I. Investigation into the neural correlates of emotional augmentation ofclinical pain. Neuroimage. 2008; 40: 759-66.

21. McNamara RK, Lotrich FE. Elevated immune-inflammatory signaling inmood disorders: a new therapeutic target? Expert Rev Neurother. 2012; 12: 1143-61.

22. Brunello N, Alboni S, Capone G, Benatti C, Blom JM, Tascedda F, et al. Acetylsalicylicacid accelerates the antidepressant effect of fluoxetine in the chronic escapedeficit model of depression. Int Clin Psychopharmacol. 2006; 21: 219-25.

23. King NJ, Thomas SR. Molecules in focus: 
indoleamine 2,3-dioxygenase. Int J Biochem Cell Biol. 2007; 39: 2167-72.

24. Krueger JM, Clinton JM, Winters BD, Zielinski MR, Taishi P, Jewett KA, et al. Involvement of cytokines in slow wave sleep. Prog Brain Res. 2011; 193: 39-47.

25. Kettenmann H, Kirchhoff F, Verkhratsky A. Microglia: new roles for thesynaptic stripper. Neuron. 2013; 77: 10-8.

26. World Health Organization. Cancer pain relief: with a guide to opioid availability. $2^{\text {nd }} \mathrm{ed}$. Geneva: World Health Organization. 1996.
27. Torta RG, Ieraci V. Pharmacological management of depression in patients with cancer: practical considerations. Drugs. 2013; 73: 1131-45.

28. Catena-Dell'Osso M, Bellantuono C, Consoli G, Baroni S, Rotella F, Marazziti D. Inflammatory and neurodegenerative pathways in depression: a new avenue for antidepressant development? Curr Med Chem. 2011; 18: 245-55.

29. Leo RJ. Pain management for psychiatrist. Arlington: American Psychiatric Publishing; 2003. 